\title{
Synthesis of some novel enzyme inhibitors and antibacterial agents derived from 5-(1-(4-tosyl)piperidin-4-yl)-1,3,4-oxadiazol-2-thiol
}

\author{
Almas Sattar ${ }^{1}$, Aziz-ur-Rehman ${ }^{1, *}$, Muhammad Athar Abbasi ${ }^{1}$, Sabahat Zahra Siddiqui ${ }^{1}$, Shahid \\ Rasool $^{1}$, Irshad Ahmad ${ }^{2}$
}

${ }^{1}$ Department of Chemistry, Government College University, Lahore-54000, Pakistan. ${ }^{2}$ Department of Pharmacy, The Islamia University of Bahawalpur, Bahawalpur, Pakistan

\begin{abstract}
Keeping in mind the pharmacological importance of the 1,3,4-oxadiazole moiety, a series of new $S$-substituted derivatives, 5a-h, of 5-(1-(4-tosyl)piperidin-4-yl)-1,3,4-oxadiazol-2-thiol (3) were synthesized. The reaction of $p$-toluenesulfonyl chloride (a) and ethyl isonipecotate (b) produced ethyl 1-(4-tosyl)piperidin-4-carboxylate (1) which was further transformed into 1-(4-tosyl)piperidin4-carbohydrazide (2) by hydrazine hydrate in methanol. Compound 2 was refluxed with $\mathrm{CS}_{2}$ in the presence of $\mathrm{KOH}$ to synthesize 5-(1-(4-tosyl)piperidin-4-yl)-1,3,4-oxadiazol-2-thiol (3). The desired compounds, $\mathbf{5 a - h}$, were synthesized by stirring $\mathbf{3}$ with aralkyl halides, $\mathbf{4 a - h}$, in DMF using NaH as an activator. The structures of synthesized compounds were elucidated by ${ }^{1} \mathrm{H}-\mathrm{NMR}$, IR and EI-MS spectral studies. These compounds were further evaluated for enzyme inhibitory activity against lipoxygenase and alpha-glucosidase, along with antibacterial activity against Gram-negative and Gram-positive bacteria.
\end{abstract}

Uniterms: 1,3,4-Oxadiazole/antibacterial activity. 1,3,4-Oxadiazole/enzyme inhibitory activity. Isonipecotate. Sulfonamide.

\begin{abstract}
Tendo em vista a importância farmacológica da porção 1,3,4-oxadiazol, sintetizou-se uma série de novos derivados $S$-substituídos, 5a-h, de 5-(1-(4-tosi)piperidin-4-il)-1,3,4-oxadiazol-2-tiol (3). A reação do cloreto de $p$-toluenossulfonila (a), com isonipecotato de (b) etila forneceu 1-(4-tosil)piperidin-4carboxilato de metila (1), que foi, em seguida, transformado em 1-(4-tosil)piperidin-4-carbo-hidrazida (2) por reação com hidrato de hidrazina em metanol. O composto 2 foi submetido a refluxo com $\mathrm{CS}_{2}$ na presença de $\mathrm{KOH}$ para se obter 5-(1-(4-tosil)piperidin-4-il)-1,3,4-oxadiazol-2-tiol (3). Os compostos desejados, $\mathbf{5} \mathbf{a}-\mathbf{h}$, foram obtidos por agitação de $\mathbf{3}$ com haletos de aralquila, $\mathbf{4 a - h}$, em DMF, na presença de $\mathrm{NaH}$. As estruturas dos compostos sintetizados foram elucidadas através de análise dos espectros de ${ }^{1} \mathrm{H}-\mathrm{MNR}$, IR e EI-MS. Estes compostos foram, ainda, avaliados quanto à inibição das enzimas lipoxigenase e alfa-glucosidase, juntamente com a atividade antibacteriana contra bactérias Gram positivas e Gram negativas.
\end{abstract}

Unitermos: 1,3,4-Oxadiazol/atividade antibacteriana. 1,3,4-Oxadiazol/inibição enzimática. Isonipecotato. Sulfonamida.

\section{INTRODUCTION}

Scientists have been working to develop new drugs for combating and controlling different diseases for decades. This task has been taken up by chemists and pharmacologists to design and synthesize novel drugs to treat various disorders and diseases in plants, animals and

*Correspondence: Aziz-ur-Rehman. Department of Chemistry. Government College University. Lahore-54000. Pakistan. E-mail: azizryk@yahoo.com, rehman@gcu.edu.pk especially humans, resulting in the emergence of various drugs. Sulfonamide, oxadiazole and piperidine derivatives with significant pharmacological activities have been introduced.

1,3,4-Oxadiazoles comprise a class of heterocyclic compounds that have a wide variety of biological activities. Their derivatives show, for example, remarkable antiproliferative (El-Din et al., 2015), antihepatitis (Tan et al., 2006), antitumor (Zhang et al., 2014), anticancer (Kumar et al., 2009), antiinflammatory (Omar et al., 1996), and antibacterial (Bhardwaj et al., 2009; Li et al., 
2014; Shafi, Radhakrishnan, 1995) activities. Piperidinebearing compounds are well known for their therapeutic potential. A number of these compounds have been synthesized and evaluated for pharmacological potential (Sanchez-Sancho, Herrandón, 1998). These compounds are used to control plasma insulin and glucose levels, in cocaine abuse treatment and also as anesthetics (Nithiya et al., 2011). Sulfonamides are also potent pharmacological and therapeutic agents which are used as antibacterial agents and inhibitors of different disease-related enzymes. These compounds are also used as anticancer drugs, diuretics and hypoglycemic agents (Adger et al., 1996; Aziz-ur-Rehman et al., 2011).

As an extension of the last work by our group (Aziz-ur-Rehman et al., 2014; Nafeesa et al., 2015), the very attractive biological activities of the abovementioned classes of compounds prompted us to synthesize compounds bearing three moieties, namely 1,3,4-oxadiazole, piperidine and sulfonamide. These new compounds were evaluated for their pharmacological potential as antibacterial and anti-enzymatic agents.

\section{MATERIAL AND METHODS}

\section{General}

The melting points of synthesized compounds were determined with a Griffin and George melting point apparatus using an open capillary tube and were uncorrected. The purity of the synthesized compounds was confirmed using thin-layer chromatography (TLC), performed on aluminum plates pre-coated with silica gel G-25-UV ${ }_{254}$, carried out under different solvent systems with varying ratios of ethyl acetate and $n$-hexane to obtain a single spot, visualized using a 254-nm UV lamp. The IR spectra were recorded using the $\mathrm{KBr}$ pellet method on a Jasco-320-A spectrometer (wave number in $\mathrm{cm}^{-1}$ ). Proton nuclear magnetic resonance spectra were recorded in $\mathrm{CDCl}_{3}$ solvent on a Bruker spectrometer operating at $400 \mathrm{MHz}$. Chemical shifts are given in ppm. Mass spectra (EIMS) were recorded on a JMS-HX-110 spectrometer, with a data system.

\section{Preparation of ethyl 1-(4-tosyl)piperidin-4- carboxylate (1)}

Ethyl isonipecotate $(\mathbf{b} ; 15.0 \mathrm{mmol})$ was dissolved in $15 \mathrm{~mL}$ distilled water in a $250 \mathrm{~mL}$ round bottom (RB) flask. Then $p$-toluenesulfonyl chloride (a; $15.0 \mathrm{mmol}$ ) was added to the reaction mixture gradually over 15-20 minutes. The $\mathrm{pH}$ was maintained at 9.0 by basic aqueous solution of
$\mathrm{Na}_{2} \mathrm{CO}_{3}(5 \%)$ at room temperature. The reaction mixture was stirred for 3-4 hours and monitored using TLC. At the completion of the reaction, concentrated $\mathrm{HCl}(11 \mathrm{M}$, $2 \mathrm{~mL}$ ) was slowly added to adjust the $\mathrm{pH}$ to 6.0 and allowed to stand for 10-15 minutes. The precipitate was filtered and washed with cold distilled water to yield on drying the desired white-colored compound $\mathbf{1}$. White amorphous solid; yield: $89 \%$; M.P.: $70-72{ }^{\circ} \mathrm{C}$; molecular formula: $\mathrm{C}_{15} \mathrm{H}_{21} \mathrm{NO}_{4} \mathrm{~S}$; molecular weight: 311 ; IR (KBr, $\left.\mathrm{cm}^{-1}\right) v_{\max }: 3067$ (C-H stretching of aromatic ring), 1732 $(\mathrm{C}=\mathrm{O}$ stretching $), 1531(\mathrm{C}=\mathrm{C}$ aromatic stretching), 1335 (-SO ${ }_{2}$ - stretching), 1079 (C-O bond stretching); ${ }^{1} \mathrm{H}-\mathrm{NMR}$ (400 MHz, $\mathrm{CDCl}_{3}, \boldsymbol{\delta} / \mathrm{ppm}$ ): 7.62 (d, $J=8.0 \mathrm{~Hz}, 2 \mathrm{H}, \mathrm{H}-2$ " \& H-6"), 7.32 (d, $J=8.0 \mathrm{~Hz}, 2 \mathrm{H}, \mathrm{H}-3$ " \& H-5”), 3.98 (q, $\left.J=7.2 \mathrm{~Hz}, 2 \mathrm{H}, \mathrm{O}-\mathrm{CH}_{2}\right), 3.71-3.68\left(\mathrm{~m}, 2 \mathrm{H}, \mathrm{H}_{e}-2^{\prime}\right.$ \& $\left.\mathrm{H}_{e}-6^{\prime}\right)$, 2.73-2.62 (m, 1H, H-4'), 2.54-2.48 ( $\left.\mathrm{m}, 2 \mathrm{H}, \mathrm{H}_{-}-2^{\prime} \& \mathrm{H}_{-}-6^{\prime}\right)$, 2.42 (s, 3H, $\mathrm{CH}_{3}-4$ '), 2.10-2.08 (m, 2H, $\mathrm{H}_{e}-3$ ' \& $\mathrm{H}_{e}-5$ '), 1.60-1.86 (m, 2H, $\left.\mathrm{H}_{a}-3^{\prime} \& \mathrm{H}_{a}-5^{\prime}\right), 1.15$ ( $\mathrm{t}, J=7.2 \mathrm{~Hz}$, $\left.\mathrm{CH}_{3}\right)$; EIMS $(\mathrm{m} / z): 311[\mathrm{M}]^{+}, 266\left[\mathrm{C}_{13} \mathrm{H}_{16} \mathrm{NO}_{3} \mathrm{~S}\right]^{+}, 238$ $\left[\mathrm{C}_{12} \mathrm{H}_{16} \mathrm{NO}_{2} \mathrm{~S}\right]^{+}, 184\left[\mathrm{C}_{8} \mathrm{H}_{10} \mathrm{NO}_{2} \mathrm{~S}\right]^{+}, 170\left[\mathrm{C}_{7} \mathrm{H}_{8} \mathrm{NO}_{2} \mathrm{~S}\right]^{+}, 155$ $\left[\mathrm{C}_{7} \mathrm{H}_{7} \mathrm{O}_{2} \mathrm{~S}\right]^{+}, 91\left[\mathrm{C}_{7} \mathrm{H}_{7}\right]^{+}$.

\section{Preparation of 1-(4-tosyl)piperidin-4- carbohydrazide (2)}

Ethyl 1-(4-tosyl)piperidin-4-carboxylate (1; $13.0 \mathrm{mmol}$ ) was dissolved in $20 \mathrm{~mL}$ methanol in a $250 \mathrm{~mL}$ RB flask. Hydrazine hydrate $(80 \%, 10 \mathrm{~mL})$ was added dropwise to the reaction mixture, which was then refluxed for 4-5 hours. Completion of the reaction was determined by TLC. At the end of the reaction, excess solvent was evaporated to yield a white crystalline product, compound 2, which was washed with cold distilled water and dried. White crystalline solid; yield: $91 \%$; M.P.: $128-130{ }^{\circ} \mathrm{C}$; molecular formula: $\mathrm{C}_{13} \mathrm{H}_{19} \mathrm{~N}_{3} \mathrm{O}_{3} \mathrm{~S}$; molecular weight: 297; IR (KBr, cm $\left.{ }^{-1}\right) v_{\max }: 3348$ (N-H stretching), 3063 (C-H stretching of aromatic ring), $1682(\mathrm{C}=\mathrm{O}$ stretching $)$, $1534\left(\mathrm{C}=\mathrm{C}\right.$ aromatic stretching), 1339 ( $-\mathrm{SO}_{2}$ - stretching); ${ }^{1} \mathrm{H}-\mathrm{NMR}\left(400 \mathrm{MHz}, \mathrm{CDCl}_{3}, \boldsymbol{\delta} / \mathrm{ppm}\right): 7.61(\mathrm{~d}, J=8.0 \mathrm{~Hz}$, 2H, H-2" \& H-6"), 7.33 (d, $J=8.0$ Hz, 2H, H-3" \& H-5"), 3.72-3.69 (m, 2H, $\left.\mathrm{H}_{e}-2^{\prime} \& \mathrm{H}_{e}-6^{\prime}\right)$, 2.73-2.62 (m, 1H, H-4'), 2.53-2.49 (m, 2H, $\mathrm{H}_{a}-2$ ' \& $\mathrm{H}_{a}-6$ '), 2.42 (s, 3H, $\mathrm{CH}_{3}-4^{\prime}$ '), 2.12-2.10 (m, 2H, $\left.\mathrm{H}_{e}-3^{\prime} \& \mathrm{H}_{e}-5^{\prime}\right), 1.58-1.84$ (m, 2H, $\mathrm{H}_{a}-3$ ' $\left.\& \mathrm{H}_{a}-5^{\prime}\right)$; $\operatorname{EIMS}(\mathrm{m} / \mathrm{z}): 297[\mathrm{M}]^{+}, 266\left[\mathrm{C}_{13} \mathrm{H}_{16} \mathrm{NO}_{3} \mathrm{~S}\right]^{+}, 238$ $\left[\mathrm{C}_{12} \mathrm{H}_{16} \mathrm{NO}_{2} \mathrm{~S}\right]^{+}, 184\left[\mathrm{C}_{8} \mathrm{H}_{10} \mathrm{NO}_{2} \mathrm{~S}\right]^{+}, 170\left[\mathrm{C}_{7} \mathrm{H}_{8} \mathrm{NO}_{2} \mathrm{~S}\right]^{+}, 155$ $\left[\mathrm{C}_{7} \mathrm{H}_{7} \mathrm{O}_{2} \mathrm{~S}\right]^{+}, 91\left[\mathrm{C}_{7} \mathrm{H}_{7}\right]^{+}$.

\section{Preparation of 5-(1-(4-tosyl)piperidin-4-yl)-1,3,4- oxadiazol-2-thiol (3)}

1-(4-Tosyl)piperidin-4-carbohydrazide (2; 
$11.0 \mathrm{mmol})$ was dissolved in methanol $(30 \mathrm{~mL})$ in a 250 -mL RB flask. Potassium hydroxide $(11.0 \mathrm{mmol})$ and carbon disulfide $(22.0 \mathrm{mmol})$ were added to the reaction mixture. The reaction mixture was refluxed for 5-6 hours and monitored for completion with TLC. At the completion of reaction, chilled distilled water ( 50 $\mathrm{mL}$ ) was added to the reaction mixture, which was then acidified to $\mathrm{pH}$ 2-3 with dilute hydrochloric acid to yield a solid precipitate. An off-white precipitate of product 3 was filtered, washed with cold distilled water and dried. White amorphous solid; yield: $87 \%$; M.P.: $230-233{ }^{\circ} \mathrm{C}$; molecular formula: $\mathrm{C}_{14} \mathrm{H}_{17} \mathrm{~N}_{3} \mathrm{O}_{3} \mathrm{~S}_{2}$; molecular weight: 339 ; IR $\left(\mathrm{KBr}, \mathrm{cm}^{-1}\right) v_{\max }: 3067(\mathrm{C}-\mathrm{H}$ stretching of aromatic ring), 2522 ( $\mathrm{S}-\mathrm{H}$ bond stretching), $1641(\mathrm{C}=\mathrm{N}$ stretching of oxadiazole ring), 1541 ( $\mathrm{C}=\mathrm{C}$ aromatic stretching), 1345 (-SO ${ }_{2}$ - stretching), $1249 \& 1079$ (C-O-C bond stretching); ${ }^{1} \mathrm{H}-\mathrm{NMR}\left(400 \mathrm{MHz}, \mathrm{CDCl}_{3}, \delta / \mathrm{ppm}\right): 7.63(\mathrm{~d}, J=8.0 \mathrm{~Hz}$, 2H, H-2" \& H-6"), 7.32 (d, J=8.0 Hz, 2H, H-3" \& H-5"), 3.71-3.68 (m, 2H, $\mathrm{H}_{e}-2^{\prime} \& \mathrm{H}_{e}-6$ '), 2.74-2.63 (m, 1H, H-4'), 2.54-2.48 (m, 2H, $\mathrm{H}_{a}-2$ ' \& $\mathrm{H}_{a}-6$ '), 2.42 (s, 3H, $\mathrm{CH}_{3}-4$ '”), 2.10-2.08 (m, 2H, $\left.\mathrm{H}_{e}-3^{\prime} \& \mathrm{H}_{e}-5^{\prime}\right), 1.59-1.85$ ( $\mathrm{m}, 2 \mathrm{H}, \mathrm{H}_{a}-3^{\prime}$ $\left.\& \mathrm{H}_{a}-5^{\prime}\right)$; $\operatorname{EIMS}(\mathrm{m} / \mathrm{z}): 339[\mathrm{M}]^{+}, 266\left[\mathrm{C}_{13} \mathrm{H}_{16} \mathrm{NO}_{3} \mathrm{~S}\right]^{+}, 238$ $\left[\mathrm{C}_{12} \mathrm{H}_{16} \mathrm{NO}_{2} \mathrm{~S}\right]^{+}, 184\left[\mathrm{C}_{8} \mathrm{H}_{10} \mathrm{NO}_{2} \mathrm{~S}\right]^{+}, 170\left[\mathrm{C}_{7} \mathrm{H}_{8} \mathrm{NO}_{2} \mathrm{~S}\right]^{+}, 155$ $\left[\mathrm{C}_{7} \mathrm{H}_{7} \mathrm{O}_{2} \mathrm{~S}\right]^{+}, 91\left[\mathrm{C}_{7} \mathrm{H}_{7}\right]^{+}$.

\section{General procedure for the synthesis of $S$-substituted derivatives of 3 (5a-h)}

A calculated amount of 5-(1-(4-tosyl)piperidin-4yl)-1,3,4-oxadiazol-2-thiol $(3 ; 8.0 \mathrm{mmol})$ was added to a 50-mL RB flask. $N, N$-Dimethylformamide (DMF, $10 \mathrm{~mL}$ ) was added to dissolve $\mathbf{3}$, followed by the addition of sodium hydride $(8.0 \mathrm{mmol})$ to the reaction mixture at room temperature and stirring for 0.5 hour. The aralkyl halides 4a-h were then added in an equimolar ratio to $\mathbf{3}$, and the reaction was further stirred for 3-4 hours. The progress of reaction was monitored with TLC until a single spot was obtained. Distilled water was added to the reaction mixture, and products $\mathbf{5 a - h}$ were recovered by filtration, which were then washed and dried.

\section{4-(2-(Benzylthio)-1,3,4-oxadiazol-5-yl)-1-(4-tosyl) piperidine (5a)}

White crystalline solid; yield: $85 \%$; M.P.: 128 $130{ }^{\circ} \mathrm{C}$; molecular formula: $\mathrm{C}_{21} \mathrm{H}_{23} \mathrm{~N}_{3} \mathrm{O}_{3} \mathrm{~S}_{2}$; molecular weight: 429; IR ( $\left.\mathrm{KBr}, \mathrm{cm}^{-1}\right) \boldsymbol{v}_{\max }: 3047$ (C-H stretching of aromatic ring), $1649(\mathrm{C}=\mathrm{N}$ stretching of oxadiazole ring), 1544 ( $\mathrm{C}=\mathrm{C}$ aromatic stretching), $1339\left(-\mathrm{SO}_{2}\right.$ - stretching), $1248 \& 1079$ (C-O-C bond stretching); ${ }^{1} \mathrm{H}-\mathrm{NMR}$ (400 $\left.\mathrm{MHz}, \mathrm{CDCl}_{3}, \delta / \mathrm{ppm}\right): 7.62$ (d, $J=8.0 \mathrm{~Hz}, 2 \mathrm{H}, \mathrm{H}-2$ '” \& H-6"), 7.31 (d, $J=7.6$ Hz, 2H, H-3" \& H-5"), 7.38-7.26 (m, 5H, H-2" " to H-6"'), 4.40(s, 2H, $\mathrm{CH}_{2}-7$ "'), 3.663.63 (m, 2H, $\mathrm{H}_{e}-2^{\prime} \& \mathrm{H}_{e}-6^{\prime}$ ), 2.84-2.79 (m, 1H, H-4'), 2.60-2.53 (m, 2H, $\mathrm{H}_{a}-2$ ' \& $\mathrm{H}_{a}-6$ '), 2.42 (s, 3H, $\mathrm{CH}_{3}-4$ '”), 2.10-2.05 (m, 2H, $\left.\mathrm{H}_{e}-3^{\prime} \& \mathrm{H}_{e}-5^{\prime}\right), 1.98-1.89$ (m, 2H, $\mathrm{H}_{a}-$ 3' \& $\mathrm{H}_{a}-5$ '); $\operatorname{EIMS}(\mathrm{m} / \mathrm{z})$ : $429[\mathrm{M}]^{+}, 266\left[\mathrm{C}_{13} \mathrm{H}_{16} \mathrm{NO}_{3} \mathrm{~S}^{+}\right.$, $238\left[\mathrm{C}_{12} \mathrm{H}_{16} \mathrm{NO}_{2} \mathrm{~S}\right]^{+}, 184\left[\mathrm{C}_{8} \mathrm{H}_{10} \mathrm{NO}_{2} \mathrm{~S}\right]^{+}, 170$ $\left[\mathrm{C}_{7} \mathrm{H}_{8} \mathrm{NO}_{2} \mathrm{~S}\right]^{+}, 155\left[\mathrm{C}_{7} \mathrm{H}_{7} \mathrm{O}_{2} \mathrm{~S}\right]^{+}, 91\left[\mathrm{C}_{7} \mathrm{H}_{7}\right]^{+}, 83\left[\mathrm{C}_{5} \mathrm{H}_{9} \mathrm{~N}\right]^{+}$, $51\left[\mathrm{C}_{4} \mathrm{H}_{3}\right]^{+}$.

\section{4-(2-(4-Fluorobenzylthio)-1,3,4-oxadiazol-5-yl)-1-(4- tosyl)piperidine (5b)}

White amorphous solid; yield: 83\%; M.P.: 135$137{ }^{\circ} \mathrm{C}$; molecular formula: $\mathrm{C}_{21} \mathrm{H}_{22} \mathrm{FN}_{3} \mathrm{O}_{3} \mathrm{~S}_{2}$; molecular weight: 447 ; IR $\left(\mathrm{KBr}, \mathrm{cm}^{-1}\right) v_{\text {max }}: 3055(\mathrm{C}-\mathrm{H}$ stretching of aromatic ring), $1658(\mathrm{C}=\mathrm{N}$ stretching of oxadiazole ring), $1554(\mathrm{C}=\mathrm{C}$ aromatic stretching $), 1361$ ( $-\mathrm{SO}_{2}$ - stretching), $1256 \& 1087$ (C-O-C bond stretching), 1178 (C-F bond stretching); ${ }^{1} \mathrm{H}-\mathrm{NMR}\left(400 \mathrm{MHz}, \mathrm{CDCl}_{3}, \delta / \mathrm{ppm}\right): 7.62$ (d, $J=8.0 \mathrm{~Hz}, 2 \mathrm{H}, \mathrm{H}-2$ " \& H-6"), 7.35 (dist. dd, $J_{\left(\mathrm{b}, \mathrm{a} \& \mathrm{~b},{ }^{19}\right.}$, $\left.{ }^{2}\right)$ 8.8, 5.2 Hz, 2H, H-2" " \& H-6"'), 7.31 (d, $J=8.0 \mathrm{~Hz}, 2 \mathrm{H}$, H-3" \& H-5"), 6.98 (br. t, $J_{\left(\mathrm{a}, \mathrm{b} \& \mathrm{a},{ }^{19}\right)}=8.8 \mathrm{~Hz}, 2 \mathrm{H}_{\mathrm{a}, \mathrm{H}} \mathrm{H}-3$ ", \& H-5" "), 4.37 (s, 2H, $\mathrm{CH}_{2}-7$ "'), 3.67-3.64 (m, 2H, $\mathrm{H}_{e}-$ 2' \& $\mathrm{H}_{e}-6$ '), 2.85-2.78 (m, 1H, H-4'), 2.59-2.53 (m, 2H, $\mathrm{H}_{a}-2$ ' \& $\mathrm{H}_{a}-6$ '), 2.42 (s, 3H, $\mathrm{CH}_{3}-4$ ') $)$ 2.10-2.06 (m, 2H, $\left.\mathrm{H}_{e}-3^{\prime} \& \mathrm{H}_{e}-5^{\prime}\right)$, 1.98-1.89 (m, 2H, $\left.\mathrm{H}_{a}-3^{\prime} \& \mathrm{H}_{a}-5^{\prime}\right)$; EIMS $(\mathrm{m} / z): 447[\mathrm{M}]^{+}, 266\left[\mathrm{C}_{13} \mathrm{H}_{16} \mathrm{NO}_{3} \mathrm{~S}\right]^{+}, 238\left[\mathrm{C}_{12} \mathrm{H}_{16} \mathrm{NO}_{2} \mathrm{~S}\right]^{+}$, $184\left[\mathrm{C}_{8} \mathrm{H}_{10} \mathrm{NO}_{2} \mathrm{~S}\right]^{+}, 170\left[\mathrm{C}_{7} \mathrm{H}_{8} \mathrm{NO}_{2} \mathrm{~S}\right]^{+}, 155\left[\mathrm{C}_{7} \mathrm{H}_{7} \mathrm{O}_{2} \mathrm{~S}\right]^{+}$ $109\left[\mathrm{C}_{7} \mathrm{H}_{6} \mathrm{~F}\right]^{+}, 91\left[\mathrm{C}_{7} \mathrm{H}_{7}\right]^{+}, 90\left[\mathrm{C}_{7} \mathrm{H}_{6}\right]^{+}, 83\left[\mathrm{C}_{5} \mathrm{H}_{9} \mathrm{~N}\right]^{+}, 83$ $\left[\mathrm{C}_{5} \mathrm{H}_{4} \mathrm{~F}\right]^{+}, 64\left[\mathrm{C}_{5} \mathrm{H}_{4}\right]^{+}, 51\left[\mathrm{C}_{4} \mathrm{H}_{3}\right]^{+}$.

\section{4-(2-(2-Chlorobenzylthio)-1,3,4-oxadiazol-5-yl)-1-(4- tosyl)piperidine (5c)}

Fluffy white amorphous solid; yield: 78\%; M.P.: 205$207{ }^{\circ} \mathrm{C}$; molecular formula: $\mathrm{C}_{21} \mathrm{H}_{22} \mathrm{ClN}_{3} \mathrm{O}_{3} \mathrm{~S}_{2}$; molecular weight: 463; IR $\left(\mathrm{KBr}, \mathrm{cm}^{-1}\right) v_{\max }: 3043$ (C-H stretching of aromatic ring), $1641(\mathrm{C}=\mathrm{N}$ stretching of oxadiazole ring), $1545\left(\mathrm{C}=\mathrm{C}\right.$ aromatic stretching), 1357 ( $-\mathrm{SO}_{2}$ - stretching), $1245 \& 1082$ (C-O-C bond stretching), 713 (C-Cl bond stretching); ${ }^{1} \mathrm{H}-\mathrm{NMR}\left(400 \mathrm{MHz}, \mathrm{CDCl}_{3}, \delta / \mathrm{ppm}\right): 7.62$ (d, $J=8.0 \mathrm{~Hz}, 2 \mathrm{H}, \mathrm{H}-2$ " \& H-6"), 7.53 (d, $J=6.8 \mathrm{~Hz}, 1 \mathrm{H}$, H-3"'), 7.37 (dd, $J=7.6,1.2 \mathrm{~Hz}, 1 \mathrm{H}, \mathrm{H}-6 "$ "), 7.31 (d, $J$ $=8.0 \mathrm{~Hz}, 2 \mathrm{H}, \mathrm{H}-3$ " \& H-5"), 7.24-7.17 (m, 2H H-4" " \& H-5 "'), 4.52 (s, 2H, $\mathrm{CH}_{2}-7$ '"'), 3.67-3.64 (m, 2H, $\mathrm{H}_{e}-2$ ' \& $\left.\mathrm{H}_{e}-6^{\prime}\right), 2.84-2.77$ (m, 1H, H-4'), 2.59-2.54 (m, 2H, $\mathrm{H}_{a}-2^{\prime}$ $\& \mathrm{H}_{a}-6$ '), 2.42 (s, 3H, $\mathrm{CH}_{3}-4$ '), 2.10-2.06 (m, 2H, $\mathrm{H}_{e}-3$ ' \& $\mathrm{H}_{e}-5^{\prime}$ ) $), 1.98-1.90$ (m, 2H, $\left.\mathrm{H}_{a}-3^{\prime} \& \mathrm{H}_{a}-5^{\prime}\right)$; EIMS ( $\left.\mathrm{m} / \mathrm{z}\right): 465$ $[\mathrm{M}+2]^{+}, 463[\mathrm{M}]^{+}, 266\left[\mathrm{C}_{13} \mathrm{H}_{16} \mathrm{NO}_{3} \mathrm{~S}\right]^{+}, 238\left[\mathrm{C}_{12} \mathrm{H}_{16} \mathrm{NO}_{2} \mathrm{~S}\right]^{+}$, $184\left[\mathrm{C}_{8} \mathrm{H}_{10} \mathrm{NO}_{2} \mathrm{~S}\right]^{+}, 170\left[\mathrm{C}_{7} \mathrm{H}_{8} \mathrm{NO}_{2} \mathrm{~S}\right]^{+}, 155\left[\mathrm{C}_{7} \mathrm{H}_{7} \mathrm{O}_{2} \mathrm{~S}\right]^{+}$, $125\left[\mathrm{C}_{7} \mathrm{H}_{6} \mathrm{Cl}\right]^{+}, 99\left[\mathrm{C}_{5} \mathrm{H}_{4} \mathrm{Cl}\right]^{+}, 90\left[\mathrm{C}_{6} \mathrm{H}_{6}\right]^{+}, 91\left[\mathrm{C}_{7} \mathrm{H}_{7}\right]^{+}, 83$ $\left[\mathrm{C}_{5} \mathrm{H}_{9} \mathrm{~N}\right]^{+}, 64\left[\mathrm{C}_{5} \mathrm{H}_{4}\right]^{+}, 51\left[\mathrm{C}_{4} \mathrm{H}_{3}\right]^{+}$. 
4-(2-(3-Chlorobenzylthio)-1,3,4-oxadiazol-5-yl)-1-(4tosyl)piperidine (5d)

Fluffy white amorphous solid; yield: 78\%; M.P.: 168-170 ${ }^{\circ} \mathrm{C}$; molecular formula: $\mathrm{C}_{21} \mathrm{H}_{22} \mathrm{ClN}_{3} \mathrm{O}_{3} \mathrm{~S}_{2}$; molecular weight: 463; IR (KBr, cm $\left.{ }^{-1}\right) v_{\max }: 3061(\mathrm{C}-\mathrm{H}$ stretching of aromatic ring $), 1654(\mathrm{C}=\mathrm{N}$ stretching of oxadiazole ring $), 1558(\mathrm{C}=\mathrm{C}$ aromatic stretching $)$, 1364 (-SO ${ }_{2}$ - stretching), $1256 \& 1089$ (C-O-C bond stretching),701 (C-Cl bond stretching); ${ }^{1} \mathrm{H}-\mathrm{NMR}$ (400 $\left.\mathrm{MHz}, \mathrm{CDCl}_{3}, \delta / \mathrm{ppm}\right): 7.63$ (d, $J=8.4 \mathrm{~Hz}, 2 \mathrm{H}, \mathrm{H}-2$ '" \& H-6"), 7.37 (s, 1H, H-2" "), 7.31 (d, J=8.4 Hz, 2H, H-3" \& H-5"), 7.28-7.22 (m, 3H H-4"' to H-6"'), 4.35 (s, 2H, $\mathrm{CH}_{2}-7^{\prime \prime}$ '), 3.67-3.64 (m, 2H, $\left.\mathrm{H}_{e}-2^{\prime} \& \mathrm{H}_{e}-6^{\prime}\right), 2.86-2.78$ (m, 1H, H-4'), 2.59-2.53 ( $\left.\mathrm{m}, 2 \mathrm{H}, \mathrm{H}_{a}-2^{\prime} \& \mathrm{H}_{a}-6^{\prime}\right), 2.42$ (s, 3H, $\mathrm{CH}_{3}-4$ ") ), 2.10-2.06 (m, 2H, $\mathrm{H}_{e}-3$ ' \& $\mathrm{H}_{e}-5$ '), 1.981.89 (m, 2H, $\left.\mathrm{H}_{a}-3^{\prime} \& \mathrm{H}_{a}-5^{\prime}\right)$; EIMS ( $\left.\mathrm{m} / \mathrm{z}\right): 465[\mathrm{M}+2]^{+}, 463$ $[\mathrm{M}]^{+}, 266\left[\mathrm{C}_{13} \mathrm{H}_{16} \mathrm{NO}_{3} \mathrm{~S}\right]^{+}, 238\left[\mathrm{C}_{12} \mathrm{H}_{16} \mathrm{NO}_{2} \mathrm{~S}\right]^{+}, 184$ $\left[\mathrm{C}_{8} \mathrm{H}_{10} \mathrm{NO}_{2} \mathrm{~S}\right]^{+}, 170\left[\mathrm{C}_{7} \mathrm{H}_{8} \mathrm{NO}_{2} \mathrm{~S}\right]^{+}, 155\left[\mathrm{C}_{7} \mathrm{H}_{7} \mathrm{O}_{2} \mathrm{~S}\right]^{+}, 125$ $\left[\mathrm{C}_{7} \mathrm{H}_{6} \mathrm{Cl}\right]^{+}, 99\left[\mathrm{C}_{5} \mathrm{H}_{4} \mathrm{Cl}\right]^{+}, 90\left[\mathrm{C}_{6} \mathrm{H}_{6}\right]^{+}, 91\left[\mathrm{C}_{7} \mathrm{H}_{7}\right]^{+}, 83$ $\left[\mathrm{C}_{5} \mathrm{H}_{9} \mathrm{~N}\right]^{+}, 64\left[\mathrm{C}_{5} \mathrm{H}_{4}\right]^{+}, 51\left[\mathrm{C}_{4} \mathrm{H}_{3}\right]^{+}$.

\section{4-(2-(4-Chlorobenzylthio)-1,3,4-oxadiazol-5-yl)-1-(4- tosyl)piperidine (5e)}

White amorphous solid; yield: 85\%; M.P.: 210 $212{ }^{\circ} \mathrm{C}$; molecular formula: $\mathrm{C}_{21} \mathrm{H}_{22} \mathrm{ClN}_{3} \mathrm{O}_{3} \mathrm{~S}_{2}$; molecular weight: 463; IR $\left(\mathrm{KBr}, \mathrm{cm}^{-1}\right) v_{\max }: 3049$ (C-H stretching of aromatic ring), $1639(\mathrm{C}=\mathrm{N}$ stretching of oxadiazole ring), 1543 ( $\mathrm{C}=\mathrm{C}$ aromatic stretching), 1356 ( $-\mathrm{SO}_{2}$ - stretching), $1243 \& 1078$ (C-O-C bond stretching), 699 (C-Cl bond stretching); ${ }^{1} \mathrm{H}-\mathrm{NMR}\left(400 \mathrm{MHz}, \mathrm{CDCl}_{3}, \delta / \mathrm{ppm}\right): 7.62$ (d, $J=8.4 \mathrm{~Hz}, 2 \mathrm{H}, \mathrm{H}-2$ " \& H-6"), 7.27 (d, $J=8.4 \mathrm{~Hz}, 2 \mathrm{H}$, H-3" \& H-5"), 7.33-7.24 (m, 4H,H-2"”, H-3" ", H-5" \& H-6"'), 4.35 (s, 2H, $\mathrm{CH}_{2}-7$ '"'), 3.67-3.64 (m, 2H, $\mathrm{H}_{e}-2$ ' \& $\mathrm{H}_{e}-6$ '), 2.84-2.79 (m, 1H, H-4'), 2.59-2.53 (m, 2H, $\mathrm{H}_{a}-2^{\prime}$ \& $\mathrm{H}_{a}-6$ '), 2.42 (s, 3H, $\mathrm{CH}_{3}-4$ '”), 2.10-2.05 (m, 2H, $\mathrm{H}_{e}-3$ ' \& $\left.\mathrm{H}_{e}-5^{\prime}\right), 1.98-1.88$ (m, 2H, $\mathrm{H}_{a}-3^{\prime} \& \mathrm{H}_{a}-5$ '); EIMS ( $\left.\mathrm{m} / \mathrm{z}\right)$ : 465 $[\mathrm{M}+2]^{+}, 463[\mathrm{M}]^{+}, 266\left[\mathrm{C}_{13} \mathrm{H}_{16} \mathrm{NO}_{3} \mathrm{~S}\right]^{+}, 238\left[\mathrm{C}_{12} \mathrm{H}_{16} \mathrm{NO}_{2} \mathrm{~S}\right]^{+}$, $184\left[\mathrm{C}_{8} \mathrm{H}_{10} \mathrm{NO}_{2} \mathrm{~S}\right]^{+}, 170\left[\mathrm{C}_{7} \mathrm{H}_{8} \mathrm{NO}_{2} \mathrm{~S}\right]^{+}, 155\left[\mathrm{C}_{7} \mathrm{H}_{7} \mathrm{O}_{2} \mathrm{~S}\right.$, $125\left[\mathrm{C}_{7} \mathrm{H}_{6} \mathrm{Cl}\right]^{+}, 99\left[\mathrm{C}_{5} \mathrm{H}_{4} \mathrm{Cl}\right]^{+}, 90\left[\mathrm{C}_{6} \mathrm{H}_{6}\right]^{+}, 91\left[\mathrm{C}_{7} \mathrm{H}_{7}\right]^{+}, 83$ $\left[\mathrm{C}_{5} \mathrm{H}_{9} \mathrm{~N}\right]^{+}, 64\left[\mathrm{C}_{5} \mathrm{H}_{4}\right]^{+}, 51\left[\mathrm{C}_{4} \mathrm{H}_{3}\right]^{+}$.

\section{4-(2-(2-Bromobenzylthio)-1,3,4-oxadiazol-5-yl)-1-(4- tosyl)piperidine (5f)}

White granular amorphous solid; yield: $81 \%$; M.P.: $155-157{ }^{\circ} \mathrm{C}$; molecular formula: $\mathrm{C}_{21} \mathrm{H}_{22} \mathrm{BrN}_{3} \mathrm{O}_{3} \mathrm{~S}_{2}$; molecular weight: 507; IR ( $\left.\mathrm{KBr}, \mathrm{cm}^{-1}\right) v_{\max }: 3053(\mathrm{C}-\mathrm{H}$ stretching of aromatic ring $), 1647(\mathrm{C}=\mathrm{N}$ stretching of oxadiazole ring $), 1546(\mathrm{C}=\mathrm{C}$ aromatic stretching $), 1361$ (-SO $\mathrm{SO}_{2}$ - stretching), 1255 \& 1082 (C-O-C bond stretching), 602 (C-Br bond stretching); ${ }^{1} \mathrm{H}-\mathrm{NMR}\left(400 \mathrm{MHz}, \mathrm{CDCl}_{3}, \delta\right.$ / ppm): 7.62 (d, $J=8.0 \mathrm{~Hz}, 2 \mathrm{H}, \mathrm{H}-2$ " \& H-6"), 7.63-7.55 (m, 2H, H-3"' \& H-6" "), 7.31 (d, $J=8.0 \mathrm{~Hz}, 2 \mathrm{H}, \mathrm{H}-3$ " \& H-5"), 7.16-7.12 (m, 2H H-4" \& H-5"'), 4.53 (s, 2H, $\mathrm{CH}_{2}-7^{\prime \prime}$ '), 3.67-3.64 (m, 2H, $\mathrm{H}_{e}-2$ ' \& $\mathrm{H}_{e}-6$ '), 2.85-2.79 (m, 1H, H-4'), 2.59-2.54 (m, 2H, $\left.\mathrm{H}_{a}-2^{\prime} \& \mathrm{H}_{a}-6^{\prime}\right), 2.42$ (s, 3H, $\mathrm{CH}_{3}-4$ '), 2.10-2.07 (m, 2H, $\mathrm{H}_{e}-3$ ' \& $\mathrm{H}_{e}-5$ '), 1.98$1.92\left(\mathrm{~m}, 2 \mathrm{H}, \mathrm{H}_{-}-3^{\prime} \& \mathrm{H}_{a}-5^{\prime}\right)$; EIMS $(\mathrm{m} / z)$ : $509[\mathrm{M}+2]^{+}$, $507[\mathrm{M}]^{+}, 266\left[\mathrm{C}_{13} \mathrm{H}_{16} \mathrm{NO}_{3} \mathrm{~S}\right]^{+}, 238\left[\mathrm{C}_{12} \mathrm{H}_{16} \mathrm{NO}_{2} \mathrm{~S}\right]^{+}, 184$ $\left[\mathrm{C}_{8} \mathrm{H}_{10} \mathrm{NO}_{2} \mathrm{~S}\right]^{+}, 169\left[\mathrm{C}_{7} \mathrm{H}_{6} \mathrm{Br}\right]^{+}, 170\left[\mathrm{C}_{7} \mathrm{H}_{8} \mathrm{NO}_{2} \mathrm{~S}\right]^{+}, 155$ $\left[\mathrm{C}_{7} \mathrm{H}_{7} \mathrm{O}_{2} \mathrm{~S}\right]^{+}, 143\left[\mathrm{C}_{5} \mathrm{H}_{4} \mathrm{Br}\right]^{+}, 90\left[\mathrm{C}_{7} \mathrm{H}_{6}\right]^{+}, 91\left[\mathrm{C}_{7} \mathrm{H}_{7}\right]^{+}, 83$ $\left[\mathrm{C}_{5} \mathrm{H}_{9} \mathrm{~N}\right]^{+}, 64\left[\mathrm{C}_{5} \mathrm{H}_{4}\right]^{+}, 51\left[\mathrm{C}_{4} \mathrm{H}_{3}\right]^{+}$.

\section{4-(2-(4-Bromobenzylthio)- 1,3,4-oxadiazol-5-yl)-1-(4- tosyl)piperidine $(\mathbf{5 g})$}

Fluffy white amorphous solid; yield: 79\%; M.P.: 163-165 ${ }^{\circ} \mathrm{C}$; molecular formula: $\mathrm{C}_{21} \mathrm{H}_{22} \mathrm{BrN}_{3} \mathrm{O}_{3} \mathrm{~S}_{2}$; molecular weight: 507; IR (KBr, cm $\left.{ }^{-1}\right) v_{\text {max }}: 3070(\mathrm{C}-\mathrm{H}$ stretching of aromatic ring $), 1662(\mathrm{C}=\mathrm{N}$ stretching of oxadiazole ring), $1551(\mathrm{C}=\mathrm{C}$ aromatic stretching $), 1368$ (-SO ${ }_{2}$ - stretching), $1261 \& 1092$ (C-O-C bond stretching), 582 (C-Br bond stretching); ${ }^{1} \mathrm{H}-\mathrm{NMR}\left(400 \mathrm{MHz}, \mathrm{CDCl}_{3}\right.$, $\delta$ / ppm): 7.62 (d, $J=8.4 \mathrm{~Hz}, 2 \mathrm{H}, \mathrm{H}-2$ " \& H-6"), 7.42 (d, $J=8.4 \mathrm{~Hz}, 2 \mathrm{H}, \mathrm{H}-3$ "' ' \& H-5" '), 7.31 (d, $J=8.4 \mathrm{~Hz}, 2 \mathrm{H}$, H-3" \& H-5"), 7.26 (d, J=8.4 Hz, 2H, H-2"' \& H-6"'), 4.34 (s, 2H, $\left.\mathrm{CH}_{2}-7^{\prime \prime \prime}\right)$, 3.67-3.64 (m, 2H, $\mathrm{H}_{e}-2$ ' \& $\mathrm{H}_{e}-6$ '), 2.84-2.78 (m, 1H, H-4'), 2.59-2.52 (m, 2H, $\mathrm{H}_{a}-2^{\prime}$ \& $\mathrm{H}_{a^{-}}$ 6'), 2.42 (s, 3H, $\mathrm{CH}_{3}-4$ ') $), 2.09-2.05$ (m, $2 \mathrm{H}, \mathrm{H}_{e}-3$ ' \& $\mathrm{H}_{e^{-}}$ 5'), 1.97-1.88 (m, 2H, $\left.\mathrm{H}_{a}-3^{\prime} \& \mathrm{H}_{a}-5^{\prime}\right)$;EIMS ( $\left.\mathrm{m} / \mathrm{z}\right)$ : 509 $[\mathrm{M}+2]^{+}, 507[\mathrm{M}]^{+}, 266\left[\mathrm{C}_{13} \mathrm{H}_{16} \mathrm{NO}_{3} \mathrm{~S}\right]^{+}, 238\left[\mathrm{C}_{12} \mathrm{H}_{16} \mathrm{NO}_{2} \mathrm{~S}\right]^{+}$, $184\left[\mathrm{C}_{8} \mathrm{H}_{10} \mathrm{NO}_{2} \mathrm{~S}\right]^{+}, 169\left[\mathrm{C}_{7} \mathrm{H}_{6} \mathrm{Br}\right]^{+}, 170\left[\mathrm{C}_{7} \mathrm{H}_{8} \mathrm{NO}_{2} \mathrm{~S}\right]^{+}, 155$ $\left[\mathrm{C}_{7} \mathrm{H}_{7} \mathrm{O}_{2} \mathrm{~S}\right]^{+}, 143\left[\mathrm{C}_{5} \mathrm{H}_{4} \mathrm{Br}\right]^{+}, 90\left[\mathrm{C}_{7} \mathrm{H}_{6}\right]^{+}, 91\left[\mathrm{C}_{7} \mathrm{H}_{7}\right]^{+}, 83$ $\left[\mathrm{C}_{5} \mathrm{H}_{9} \mathrm{~N}\right]^{+}, 64\left[\mathrm{C}_{5} \mathrm{H}_{4}\right]^{+}, 51\left[\mathrm{C}_{4} \mathrm{H}_{3}\right]^{+}$.

\section{4-(2-(2-Methylbenzylthio)- 1,3,4-oxadiazol-5-yl)-1-(4- tosyl)piperidine (5h)}

White amorphous solid; yield: 83\%; M.P.: 190$192{ }^{\circ} \mathrm{C}$; molecular formula: $\mathrm{C}_{22} \mathrm{H}_{25} \mathrm{~N}_{3} \mathrm{O}_{3} \mathrm{~S}_{2}$; molecular weight: 443 ; IR $\left(\mathrm{KBr}, \mathrm{cm}^{-1}\right) v_{\text {max }}: 3067(\mathrm{C}-\mathrm{H}$ stretching of aromatic ring), $1655(\mathrm{C}=\mathrm{N}$ stretching of oxadiazole ring), $1554(\mathrm{C}=\mathrm{C}$ aromatic stretching $), 1365$ ( $-\mathrm{SO}_{2}$ - stretching), $1259 \& 1093$ (C-O-C bond stretching); ${ }^{1} \mathrm{H}-\mathrm{NMR}$ (400 $\left.\mathrm{MHz}, \mathrm{CDCl}_{3}, \delta / \mathrm{ppm}\right): 7.63$ (d, $J=8.0 \mathrm{~Hz}, 2 \mathrm{H}, \mathrm{H}-2$ " \& H-6"'), 7.32-7.30 (m, 3H, H-3", H-5" \& H-3" '), 7.187.10 (m, 3H,H-4" "to H-6" "), 4.43 (s, 2H, $\mathrm{CH}_{2}-7$ "'), 3.67-3.64 (m, 2H, $\mathrm{H}_{e}-2$ ' \& $\left.\mathrm{H}_{e}-6^{\prime}\right), 2.85-2.79(\mathrm{~m}, 1 \mathrm{H}$, H-4'), 2.60-2.55 (m, 2H, $\mathrm{H}_{a}-2^{\prime}$ \& $\left.\mathrm{H}_{a}-6^{\prime}\right), 2.42$ (s, 3H, $\mathrm{CH}_{3}-4$ "), 2.39 (s, 3H, $\mathrm{CH}_{3}-2$ "'), 2.11-2.07 (m, 2H, $\mathrm{H}_{e}-3$ ' \& $\left.\mathrm{H}_{e}-5^{\prime}\right), 1.99-1.93\left(\mathrm{~m}, 2 \mathrm{H}, \mathrm{H}_{a}-3^{\prime} \& \mathrm{H}_{a}-5^{\prime}\right)$;EIMS $(\mathrm{m} / \mathrm{z})$ : $443[\mathrm{M}]^{+}, 266\left[\mathrm{C}_{13} \mathrm{H}_{16} \mathrm{NO}_{3} \mathrm{~S}\right]^{+}, 238\left[\mathrm{C}_{12} \mathrm{H}_{16} \mathrm{NO}_{2} \mathrm{~S}\right]^{+}, 184$ $\left[\mathrm{C}_{8} \mathrm{H}_{10} \mathrm{NO}_{2} \mathrm{~S}^{+}, 170\left[\mathrm{C}_{7} \mathrm{H}_{8} \mathrm{NO}_{2} \mathrm{~S}\right]^{+}, 155\left[\mathrm{C}_{7} \mathrm{H}_{7} \mathrm{O}_{2} \mathrm{~S}\right]^{+}, 106\right.$ $\left[\mathrm{C}_{8} \mathrm{H}_{10}\right]^{+}, 91\left[\mathrm{C}_{7} \mathrm{H}_{7}\right]^{+}, 90\left[\mathrm{C}_{7} \mathrm{H}_{6}\right]^{+}, 83\left[\mathrm{C}_{5} \mathrm{H}_{9} \mathrm{~N}\right]^{+}, 65\left[\mathrm{C}_{5} \mathrm{H}_{4}\right]^{+}$, $51\left[\mathrm{C}_{4} \mathrm{H}_{3}\right]^{+}$. 


\section{Biological activity assays}

\section{Lipoxygenase inhibition assay}

Lipoxygenase activity was assayed according to a previously reported method (Baylac, Racine, 2003; Nafeesa et al., 2015). The change in absorbance was determined at $234 \mathrm{~nm}$ for all the test compounds.

\section{$\alpha$-Glucosidase inhibition assay}

$\alpha$-Glucosidase inhibitory activity was performed according to a previously reported method (Abbasi et al., 2014; Chapdelaine et al., 1978). The change in absorbance was determined at $400 \mathrm{~nm}$ for all the test compounds.

\section{Antibacterial activity assay}

The antibacterial activity was performed under aseptic conditions in sterile 96 -well microplates. The principle of the method is that as microbial cell population increases during log phase growth, there is an increase in the absorbance of the broth medium (Kaspady et al., 2009; Nafeesa et al., 2015). Ciprofloxacin was used as reference standard.

\section{Statistical analysis}

Minimum inhibitory concentration (MIC) for antibacterial activity and $\mathrm{IC}_{50}$ (concentration causing $50 \%$ inhibition) for enzyme inhibition was determined with suitable dilutions for each sample, and results were calculated using EZ-Fit software (Perrella Scientific Inc., Amherst, NH, USA). The results are presented as mean \pm SEM for triplicate determinations after statistical analysis performed with MS Excel 2010.

\section{RESULTS AND DISCUSSION}

The goal of our study was to produce $S$-substituted derivatives of oxadiazole compounds having $p$-toluene sulfonyl and piperidine moieties and also to screen them for enzyme inhibitory activity. A series of derivatives, $\mathbf{5 a - h}$, were synthesized according to the protocol given in Figure 1. The different aralkyl groups are given in Table I.

\section{Chemistry}

Compound 5a was synthesized as a white crystalline compound. The molecular formula $\mathrm{C}_{21} \mathrm{H}_{23} \mathrm{~N}_{3} \mathrm{O}_{3} \mathrm{~S}_{2}$ was established with molecular ion peak $m / z 429$ in EIMS and by proton counting using ${ }^{1} \mathrm{H}-\mathrm{NMR}$ spectra. The IR spectrum gave absorption bands at 3047, 1649, 1544, 1339,1248 and $1079 \mathrm{~cm}^{-1}$, which were assigned to $\mathrm{C}-\mathrm{H}$ (stretching of aromatic ring), $\mathrm{C}=\mathrm{N}$ (stretching of oxadiazole ring), $\mathrm{C}=\mathrm{C}$ (aromatic stretching), $-\mathrm{SO}_{2}$ (stretching), and $\mathrm{C}-\mathrm{O}-\mathrm{C}$ (stretching of oxadiazole ring), respectively. In EIMS spectra, the peak at $\mathrm{m} / \mathrm{z} 266$ showed cleavage

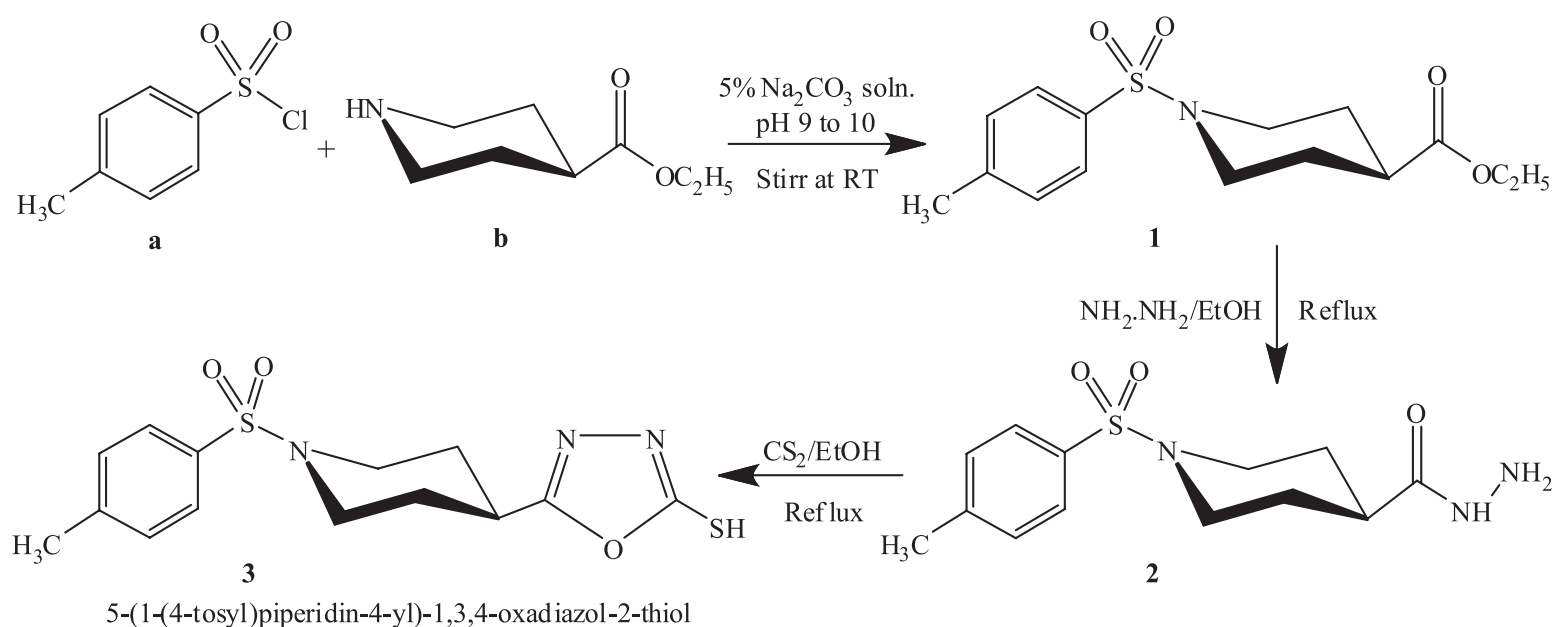

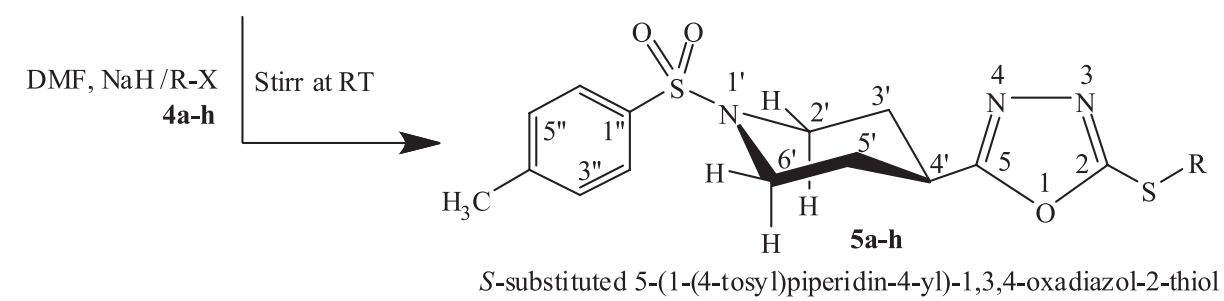

FIGURE 1 - Synthesis of $S$-substituted derivatives of 5-(1-(4-Tosyl)piperidin-4-yl)-1,3,4-oxadiazol-2-thiol (5a-h). 
TABLE I - Different aralkyl groups

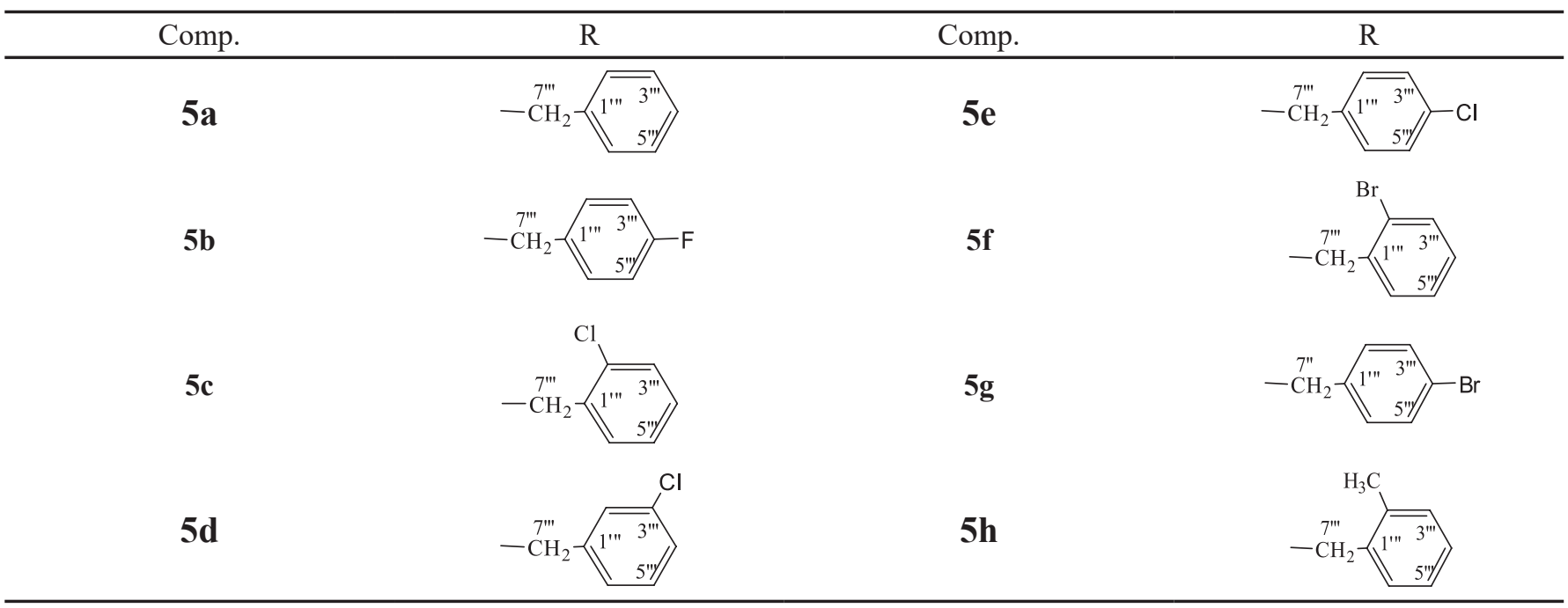

of the benzyl sulfide group along with partial cleavage of the oxadiazole ring in 5a, while the peak at $\mathrm{m} / z 155$ showed the presence of the $p$-toluenesulfonyl group and the peak at $m / z 83$ the presence of a piperidine moiety. The other prominent fragments are given in Figure 2. In the aromatic region of ${ }^{1} \mathrm{H}-\mathrm{NMR}$, signals appeared at $\delta 7.62(\mathrm{~d}$, $J=8.0 \mathrm{~Hz}, 2 \mathrm{H}, \mathrm{H}-2$ " \& H-6") and 7.31 (d, $J=7.6 \mathrm{~Hz}, 2 \mathrm{H}$, H-3" \& H-5"), which were assigned to the toluenesulfony moiety; the signals appearing at $\delta 7.38-7.26(\mathrm{~m}, 5 \mathrm{H}$, H-2" to H-6" ") were assigned to the mono-substituted benzene ring of the benzyl moiety. The signals resonating at $\delta 3.66-3.63\left(\mathrm{~m}, 2 \mathrm{H}, \mathrm{H}_{e}-2^{\prime} \& \mathrm{H}_{e}-6^{\prime}\right), 2.84-2.79(\mathrm{~m}, 1 \mathrm{H}$, H-4'), 2.60-2.53 (m, 2H, $\left.\mathrm{H}_{a}-2^{\prime} \& \mathrm{H}_{a}-6^{\prime}\right), 2.10-2.05$ (m, 2H, $\mathrm{H}_{e}-3$ ' \& $\left.\mathrm{H}_{e}-5^{\prime}\right)$ and1.98-1.89 (m, $2 \mathrm{H}, \mathrm{H}_{a}-3$ ' \& $\mathrm{H}_{a}-5$ ') were assigned to the piperidine moiety; and the signal at 4.40 (s, $2 \mathrm{H}, \mathrm{CH}_{2}-7$ "') was assigned to the methylene group of the benzyl moiety. On the basis of the above findings, the structure of 5a was determined to be 4-(2-(benzylthio)1,3,4-oxadiazol-5-yl)-1-(4-tosyl)piperidine. In a similar way, the structures of other synthesized compounds were characterized by using ${ }^{1} \mathrm{H}-\mathrm{NMR}$, IR and EIMS data.

\section{Enzyme inhibitory activity (in vitro)}

The screening of synthesized compounds against the enzymes lipoxygenase and $\alpha$-glucosidase revealed that most of the compounds were moderate to weakly moderate inhibitors of these enzymes. The experimental results are given in Table II below.

Screening of these synthesized compounds proved $\mathbf{5 b}$ to be the most active inhibitor against lipoxygenase. Other compounds showed no activity, except $\mathbf{5 d}$ against this enzyme. Only the $S$-substituted benzyl compound containing fluorine at the para position showed better activity against this enzyme. The better activity of $\mathbf{5 b}$ was attributed to the presence of a fluorine at position ' 4 ' in th ebenzyl moiety. The compounds $\mathbf{5} \mathbf{d}$ and $\mathbf{5} \mathbf{f}$ remained inactive against $\alpha$-glucosidase. $\mathbf{5 b}$ showed the highest inhibitory activity with an $\mathrm{IC}_{50}$ of $181.92 \pm 0.17 \mu \mathrm{M}$ compared to $38.25 \pm 0.12 \mu \mathrm{M}$ of acarbose, the reference standard. The high inhibitory activity of this compound could be attributed to the $S$-substituted benzyl moiety containing a highly electronegative group at the para position, which showed the best catalysis-blocking capability. The other compounds showed moderately weak inhibition, but all 4-substituted halogenated benzyl group-containing compounds had relatively better activity in a sequential order. $\mathrm{The}^{\mathrm{I}} \mathrm{C}_{50}$ values of 4-substituted halogenated benzyl groupcontaining compounds indicated that the presence of small and more electronegative atoms leads to better inhibition of the enzyme. The inhibition order of molecules containing 4-substituted halogenated benzyl group was fluorinated $>$ chlorinated $>$ brominated. The overall descending order of inhibitory activity of the synthesized molecules was as follows, $5 \mathbf{b}, 5 \mathbf{5 e}, \mathbf{5 c}, \mathbf{5 a}, \mathbf{5 g}$ and $\mathbf{5 h}$.

\section{Antibacterial activity (in vitro)}

The in vitro MIC and percentage inhibition results for antibacterial activity of the synthesized compounds against Gram-positive and Gram-negative bacteria are given in Tables III and IV, using ciprofloxacin as reference standard.

All the synthesized compounds displayed strong to moderate antibacterial activity against all the bacterial strains studied except for Staphylococcus aureus against which only $\mathbf{5} \mathbf{h}$ was moderately active. Compounds $\mathbf{5 a}$ and 


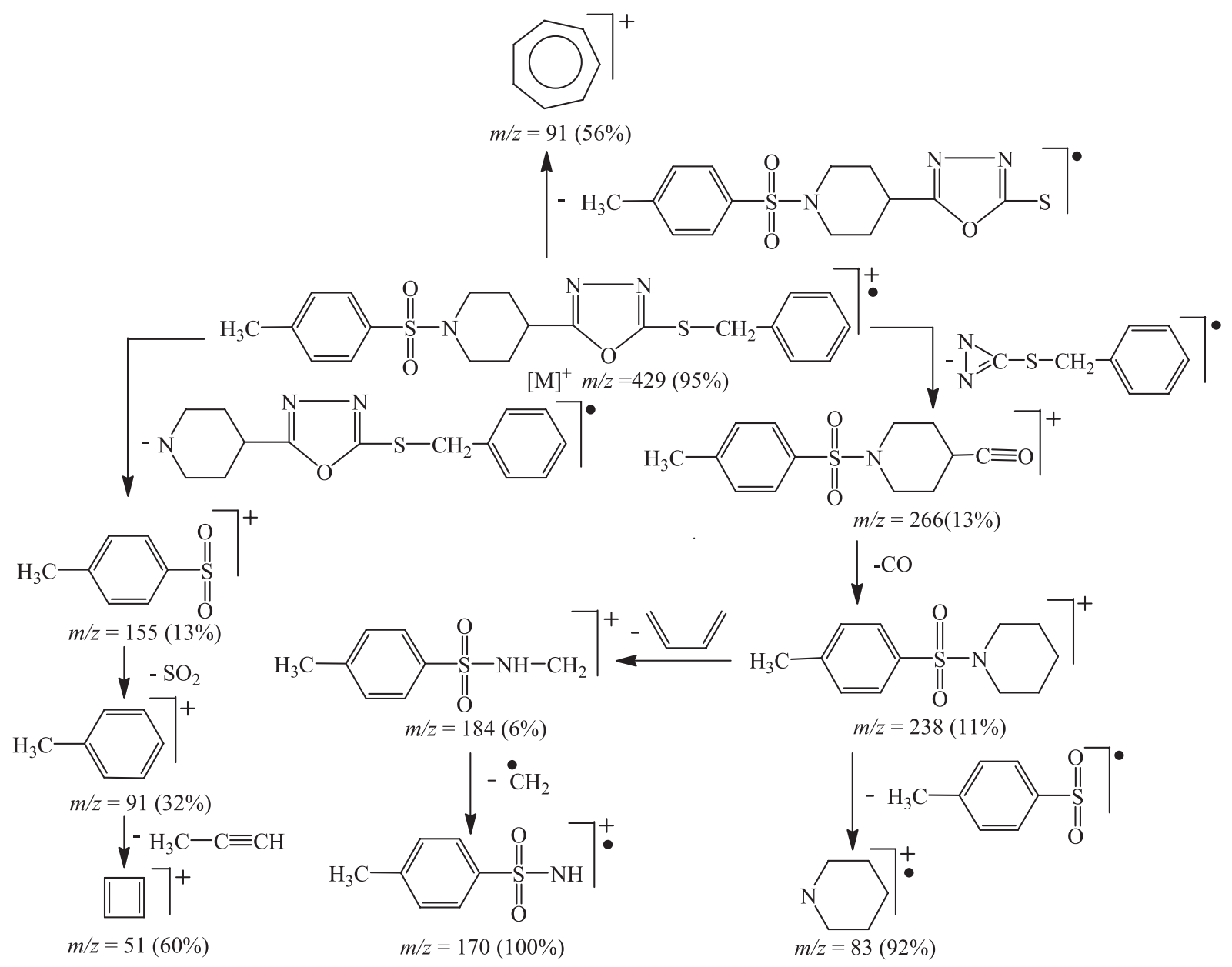

FIGURE 2 -Proposed mass fragmentation pattern of 4-(2-(benzylthio)-1,3,4-oxadiazol-5-yl)-1-(4-tosyl)piperidine (5a).

TABLE II - Enzyme inhibitory activity against lipoxygenase and $\alpha$-glucosidase enzymes

\begin{tabular}{lcccc}
\hline \multirow{2}{*}{ Compound } & \multicolumn{2}{c}{ Lipoxygenase (LOX) } & \multicolumn{2}{c}{$\boldsymbol{\alpha - G l u c o s i d a s e}$} \\
\cline { 2 - 5 } & $\begin{array}{c}\text { Inhibition (\%) } \\
\text { at } \mathbf{0 . 5} \mathbf{~ m M}\end{array}$ & $\begin{array}{c}\mathbf{I C}_{\mathbf{5 0}} \\
\boldsymbol{\mu M}\end{array}$ & $\begin{array}{c}\text { Inhibition (\%) } \\
\text { at } \mathbf{0 . 5} \mathbf{~ m M}\end{array}$ & $\begin{array}{c}\mathbf{I C}_{\mathbf{5 0}} \\
\boldsymbol{\mu M}\end{array}$ \\
\hline $\mathbf{5 a}$ & $47.81 \pm 0.06$ & - & $65.22 \pm 0.31$ & $197.98 \pm 0.11$ \\
$\mathbf{5 b}$ & $99.12 \pm 0.41$ & $78.38 \pm 0.12$ & $81.11 \pm 0.24$ & $181.92 \pm 0.17$ \\
$\mathbf{5 c}$ & $11.71 \pm 0.14$ & - & $78.14 \pm 0.31$ & $195.53 \pm 0.19$ \\
$\mathbf{5 d}$ & $56.95 \pm 0.33$ & $229.72 \pm 0.16$ & $11.33 \pm 0.25$ & - \\
$\mathbf{5 e}$ & $12.25 \pm 0.89$ & - & $83.56 \pm 0.16$ & $185.58 \pm 0.18$ \\
$\mathbf{5 f}$ & $47.43 \pm 0.56$ & - & $9.27 \pm 0.12$ & - \\
$\mathbf{5 g}$ & $11.62 \pm 0.41$ & - & $61.55 \pm 0.13$ & $313.79 \pm 0.07$ \\
$\mathbf{5 h}$ & $14.37 \pm 0.29$ & - & $84.46 \pm 0.16$ & $321.43 \pm 0.14$ \\
$\mathbf{C o n t r o l}$ & $\mathbf{9 3 . 7 9} \pm \mathbf{1 . 2 7}$ & $\mathbf{2 2 . 4 1} \pm \mathbf{1 . 3}^{\mathbf{a}}$ & $\mathbf{9 2 . 2 3} \pm \mathbf{0 . 1 4}$ & $\mathbf{3 8 . 2 5} \pm \mathbf{0 . 1 2}$ \\
\hline
\end{tabular}

Note: $\mathrm{a}=$ Baicalein, $\mathrm{b}=$ Acarbose. $\mathrm{IC}_{50}$ values (concentration at which there is $50 \%$ enzyme inhibition) of compounds were calculated using EZ-Fit Enzyme Kinetics software (Perrella Scientific Inc.)

$\mathbf{5 g}$ showed strong to moderate activity and $\mathbf{5 b}$ showed moderate activity against all strains except Staphylococcus aureus. $\mathbf{5 h}$ exhibited moderate activity against all except
Pseudomonas aeruginosa. The compounds $\mathbf{5 d}$ and $\mathbf{5 e}$ were active against only three strains, Salmonella typhi, Escherichia coli and Bacillus subtilis. 5f was active only 
TABLE III - The \% inhibition of antibacterial activity of synthesized compounds

\begin{tabular}{lccccc}
\hline \multirow{2}{*}{ Compounds } & \multicolumn{5}{c}{ \% INHIBITION } \\
\cline { 2 - 6 } & S. typhi (-) & E. coli (-) & P. aeruginosa (-) & S. aureus (+) & B. subtilis (+) \\
\hline $\mathbf{5 a}$ & $57.94 \pm 0.75$ & $61.15 \pm 0.35$ & $63.32 \pm 0.19$ & $33.43 \pm 0.10$ & $74.60 \pm 0.93$ \\
$\mathbf{5 b}$ & $55.76 \pm 0.80$ & $51.50 \pm 0.10$ & $53.93 \pm 0.89$ & $20.86 \pm 0.55$ & $56.50 \pm 1.13$ \\
$\mathbf{5 c}$ & - & - & - & - & - \\
$\mathbf{5 d}$ & $64.05 \pm 0.60$ & $70.00 \pm 1.00$ & $44.36 \pm 0.50$ & $40.28 \pm 0.55$ & $52.07 \pm 0.33$ \\
$\mathbf{5 e}$ & $72.39 \pm 0.70$ & $67.10 \pm 1.10$ & $47.02 \pm 0.56$ & $34.29 \pm 0.45$ & $58.80 \pm 0.47$ \\
$\mathbf{5 f}$ & $79.74 \pm 0.60$ & $46.70 \pm 0.65$ & $48.35 \pm 0.17$ & $29.05 \pm 0.81$ & $70.53 \pm 0.13$ \\
$\mathbf{5 g}$ & $68.55 \pm 0.09$ & $63.15 \pm 0.85$ & $64.33 \pm 0.32$ & $27.38 \pm 0.24$ & $72.57 \pm 0.81$ \\
$\mathbf{5 h}$ & $52.73 \pm 0.68$ & $57.30 \pm 0.70$ & $49.95 \pm 0.66$ & $57.14 \pm 0.29$ & $57.87 \pm 0.09$ \\
Ciprofloxacin & $\mathbf{9 1 . 0 5} \pm \mathbf{0 . 6 8}$ & $\mathbf{9 2 . 3 2} \pm \mathbf{0 . 4 2}$ & $\mathbf{9 2 . 0 2} \pm \mathbf{0 5 3}$ & $\mathbf{9 1 . 4 4} \pm \mathbf{0 . 6 4}$ & $\mathbf{9 2 . 5 0} \pm \mathbf{0 . 3 4}$ \\
\hline
\end{tabular}

TABLE IV - The MIC of antibacterial activity of synthesized compounds

\begin{tabular}{|c|c|c|c|c|c|}
\hline \multirow{2}{*}{ Compounds } & \multicolumn{5}{|c|}{ MIC $(\mu \mathrm{M})$} \\
\hline & S. typhi (-) & E. coli (-) & P. aeruginosa (-) & S. aureus (+) & B. subtilis (+) \\
\hline $5 \mathbf{a}$ & $16.42 \pm 0.10$ & $14.32 \pm 0.56$ & $12.87 \pm 0.41$ & - & $9.65 \pm 0.24$ \\
\hline $5 b$ & $16.98 \pm 0.45$ & $19.80 \pm 0.12$ & $17.97 \pm 0.15$ & - & $16.43 \pm 0.52$ \\
\hline $5 c$ & - & - & - & - & - \\
\hline $5 d$ & $11.57 \pm 0.71$ & $9.78 \pm 0.66$ & - & - & $19.80 \pm 0.18$ \\
\hline $5 e$ & $9.15 \pm 0.24$ & $10.09 \pm 0.05$ & - & - & $16.83 \pm 0.91$ \\
\hline $5 f$ & $8.24 \pm 0.90$ & - & - & - & $9.10 \pm 0.41$ \\
\hline $5 g$ & $9.69 \pm 0.16$ & $13.79 \pm 0.12$ & $11.87 \pm 0.50$ & - & $9.27 \pm 0.17$ \\
\hline $5 \mathrm{~h}$ & $17.43 \pm 0.33$ & $15.67 \pm 0.34$ & - & $16.78 \pm 0.95$ & $16.42 \pm 0.12$ \\
\hline Ciprofloxacin & $7.45 \pm 0.58$ & $7.16 \pm 0.58$ & $7.29 \pm 0.90$ & $7.80 \pm 0.19$ & $7.14 \pm 0.18$ \\
\hline
\end{tabular}

against two strains, Salmonella typhi and Bacillus subtilis, while 5c showed no activity at all. Against Salmonella typhi, the three compounds $\mathbf{5 e}, \mathbf{5} \mathbf{f}$ and $\mathbf{5 g}$ were the most efficient with MIC values of $9.15 \pm 0.24,8.24 \pm 0.90$ and $9.69 \pm 0.16 \mu \mathrm{M}$, respectively, relative to $7.45 \pm 0.58$, MIC of reference. Against Escherichia coli, the two compounds 5d and 5e showed the lowest MIC values, i.e., $9.78 \pm 0.66$ and $10.09 \pm 0.05 \mu \mathrm{M}$, respectively, relative to that of the reference, $7.16 \pm 0.58 \mu \mathrm{M}$. 5a, $\mathbf{5 f}$ and $\mathbf{5 g}$ inhibited Bacillus subtilis with MIC values of $9.65 \pm 0.24,9.10 \pm 0.41$ and $9.27 \pm 0.17 \mu \mathrm{M}$, respectively, compared to $7.14 \pm 0.18 \mu \mathrm{M}$ for the reference drug. The overview of the most active compounds revealed that the nature and position of halogen-substitution in the molecule greatly affected the biological behavior of the molecules.

\section{CONCLUSION}

The structures of the synthesized compounds are supported by spectroscopic data. From enzyme inhibition and antibacterial data, it was evident that different $S$-substituted derivatives of 5-(1-(4-tosyl)piperidin-4-yl)1,3,4-oxadiazol-2-thiol are valuable enzyme inhibitors and potential antibacterial agents. It is interesting and worth knowing that $\alpha$-glucosidase is inhibited by all 4 -halogenated benzyl $S$-substituted compounds while lipoxygenase is inhibited by only 4-fluoro benzyl $S$-substituted compounds . This information could be used for selective inhibition of alpha-glucosidase and lipoxygenase and may be of use in drug discovery programs. The antibacterial activity suggested that compounds $\mathbf{5 a}, \mathbf{5 f}$ and $\mathbf{5 g}$ against Bacillus subtilis, 5d against Escherichia coli and $\mathbf{5 e}, \mathbf{5 f}$ and $\mathbf{5 g}$ against Salmonella typhi could be considered for further in vivo evaluation by the pharmaceutic industry.

\section{ACKNOWLEDGEMENT}

The authors thank the Higher Education Commission (HEC) of Pakistan for financial assistance in supporting this project.

\section{REFERENCES}

ABBASI, M.A.; RAZA, N.; AZIZ-UR-REHMAN; RASOOL, S.; KHAN, K.M.; ASHRAF, M.; ALAM, U.; NASAR, R. In vitro enzyme inhibition study on new sulfonamide derivatives of 4-tosyl chloride. World J. Pharm. Sci., v.2, p.161-169, 2014. 
ADGER, B.; DYER, U.; HUTTON, G.; WOODS, M. Stereospecific synthesis of the anaesthetic Levobupivacaine. Tetrahedron Lett., v.37, n.35, p.6399-6402, 1996.

AZIZ-UR-REHMAN; KHALID, H.; ABBASI, M.A.; GUL, S.; AHMAD, I.; IRSHAD, S. Synthesis of potent antibacterial agents derived from 5-[1-(Phenylsulfonyl)piperidin-4-yl]1,3,4-oxadiazol-2-thiol. J. Chem. Soc. Pakistan, v.36, n.1, p.131-139, 2014.

AZIZ-UR-REHMAN; TANVEER, W.; ABBASI, M.A.; AFROZ,S.; KHAN, K.M.; ASHRAF, M.; AFZAL, I. Synthesis, characterization and biological screening of various $N$-substituted derivatives of sulfonamides. Int. J. Chem. Res., v.3, p.99-104, 2011.

BAYLAC, S.; RACINE, P. Inhibition of 5-lipoxygenase by essential oils and other natural fragrant extracts. Int. J. Aromather, v.13, n.2-3, p.138-142, 2003.

BHARDWAJ, N.; SARAF, S.K.; SHARMA, P.; KUMAR, P. Synthesis, evaluation and characterization of some 1,3,4-oxadiazole as antibacterial agent. E-J. Chem., v.6, n.4, p.1133-1138, 2009.

CHAPDELAINE, P.; TREMBLAY, R.R.; DUBE, J.Y. $p$-nitrophenol- $\alpha$-D-glucopyranoside as substrate for measurement of maltase activity in human semen. Clin. Chem., v.24, n.2, p.208-211, 1978.

EL-DIN, M.M.G.; EL-GAMAL, M.I.; ABDEL-MAKSOUD, M.S.; YOO, K.H.; OH, C.H. Synthesis and broad-spectrum anti-proliferative activity of diarylamides and diarylureas possessing 1,3,4-oxadiazole derivatives. Bioorg. Med. Chem. Lett., v.25, n.8, p.1692-1699, 2015.

KASPADY, M.; NARAYANASWAMY, V.K.; RAJU, M.; RAO, G.K. Synthesis, antibacterial activity of 2,4-disubstituted oxazoles and thiazoles as bioesters. Lett. Drug Des. Discov., v.6, n.1, p.21-28, 2009.

KUMAR, D.; SUNDAREE, S.; JOHNSON, E.O.; SHAH, K. An efficient synthesis and biological study of novel indolyl1,3,4-oxadiazoles as potent anticancer agents. Bioorg. Med. Chem. Lett., v.19, n.15, p.4492-4494, 2009.
LI, P.; SHI, L.; GAO, M.N.; YANG, X..; XUE, W.; JIN, L.H.; HU, D.Y.; SONG, B.A. Antibacterial activities against rice bacterial leaf blight and tomato bacterial wilt of 2-mercapto5-substituted-1,3,4-oxadiazole/thiadiazole derivatives. Bioorg. Med. Chem. Lett., v.25, n.3, p.481-484, 2014.

NAFEESA, K.; AZIZ-UR-REHMAN; ABBASI, M.A.; SIDDIQUI, S.Z.; RASOOL, S.; HUSSAIN, G.; AHMED, I. Synthesis and biological screening of $S$-substituted derivatives of 5-\{1-[(4-chlorophenyl)sulfonyl]-3piperidinyl\}-1,3,4-oxadiazole-2-yl sulfide. Asian J. Chem., v.27, n.6, p.2105-2108, 2015.

NITHIYA, S.; KARTHIK, N.; JAYABHARATHI, J. In vitro antioxidant activity of hindered piperidone derivatives. Int. J. Pharm. Pharm. Sci., v.3, p.254-256, 2011.

OMAR, F.A.; MAHFOUZ, N.M.; RAHMAN, M.A. Design, synthesis and anti-inflammatory activity of some 1,3,4-oxadiazole derivatives. Eur. J. Med. Chem., v.31, n.10, p.819-825, 1996.

SANCHEZ-SANCHO, F.; HERRANDÓN, B.; Short syntheses of (S)-pipecolic acid, (R)-coniine, and (S)-coniceine using biocatalytically-generated chiral building block. Tetrahedron: Asymmetry, v.9, n.11, p.1951-1965, 1998.

SHAFI, S.S.; RADHAKRISHNAN T.R. Studies on biologically active heterocycles. Part I. Synthesis and antibacterial activity of some 2,5-di-substituted-1,3,4-oxadiazole,1,3,4thiadiazole, 1,2,4-triazole and 4-thiazolidinone. Indian J. Heterocycl. Chem., v.5, p.133-138, 1995.

TAN, T.M.C.; CHENA, Y.; KONG, K.H.; BAI, J.; LI, Y.; LIM, S.G.; ANG, T.H.; LAM, Y. Synthesis and the biological evaluation of 2-benzenesulfonylalkyl-5-substitutedsulfanyl-[1,3,4]-oxadiazoles as potential anti-hepatitis B virus agents. Antivir. Res., v.71, n.1, p.7-14, 2006.

ZHANG, K.; WANG, P.; XUAN, L.N.; FU, X.Y.; JING, F.; LI, S.; LIU, Y.M.; CHEN, B.Q. Synthesis and antitumor activities of novel hybrid molecules containing 1,3,4-oxadiazole and 1,3,4-thiadiazole bearing Schiff base moiety. Bioorg. Med. Chem. Lett., v.24, n.22, p.5154-5156, 2014.

Received for publication on $28^{\text {th }}$ June 2015 Accepted for publication on $27^{\text {th }}$ October 2015 
